\title{
ELEVATION OF TESTOSTERONE AND REDUCTION OF TRANSEPIDERMAL WATER LOSS BY VIEWING A HUMOROUS FILM IN ELDERLY PATIENTS WITH ATOPIC DERMATITIS
}

\begin{abstract}
Hajime Kimata
Moriguchi-Keijinkai Hospital, Osaka Prefecture, Japan: Department of Allergy

Summary: The effect of viewing a humorous film on salivary testosterone levels and transepidermal water loss (TEWL) values on the back of the neck in 36 elderly healthy people (36 male, mean 70 years) and 36 elderly patients with atopic dermatitis (AD) (36 male, mean age 70 years) were studied. Salivary testosterone levels were decreased while TEWL values were increased in elderly patients with AD compared to those in elderly healthy people. Viewing a humorous film (The Best Bits of Mr. Bean, Universal studios, 1996) slightly, but significantly ( $<<0.05)$, elevated salivary testosterone levels and reduced TEWL values in elderly healthy people, while viewing a control non-humorous film (weather information) failed to do so. Similarly, but more pronouncedly, viewing a humorous film markedly elevated salivary testosterone levels and reduced TEWL values in elderly patients with $\mathrm{AD}$, while viewing a control non-humorous film failed to do so. These finding indicate that viewing a humorous film may be useful in the study of testosterone and TEWL, and treatment for dry skin in elderly people with or without AD.
\end{abstract}

Key words: Atopic dermatitis; Elderly people; Salivary testosterone; Transwater epidermal loss; Humorous film

\section{Introduction}

Skin barrier function was impaired and transepidermal water loss (TEWL) values were increased in patients with atopic dermatitis (AD) (2). TEWL values were also increased in elderly healthy people, thus elderly patients with AD had higher TEWL values (7). AD patients were vulnerable to stress (4), and stress impaired barrier function and increased TEWL values (5). Stress also augmented allergic responses, while relaxation by viewing a humorous film reduced allergic responses in $\operatorname{AD}$ patients $(13,14)$. In addition, inhalation of odorant with a sedative effect prevented the stress-induced delay of skin barrier recovery (8).

It was reported that serum levels of testosterone were decreased in male patients with $\mathrm{AD}$, and testosterone was involved in improvement of TEWL $(1,9)$. Stress decreased serum testosterone levels (3). Since serum testosterone levels were correlated with salivary testosterone levels, we studied whether viewing a humorous film could elevate salivary testosterone levels and reduce TEWL values in elderly AD patients (21).

\section{Materials and methods}

After obtaining informed consents from subjects, 36 elderly healthy people (36 male, mean age 70 years, range
65-76 years) and 36 elderly patients with mild AD (scoring atopic dermatitis [SCORAD] index: mean 9, 95 \% CI 7-11) (36 male, mean age 70 years, range 65-76 years) were studied (16). All of the AD patients were treated with an application of pinetar (Yoshida Pharmaceutical Co. Ltd. Tokyo, Japan) mixed with zinc oxide ointment, but without oral medication (4). The application of ointment was stopped 2 days before the study. Neither healthy people nor AD patients had any endocrinological diseases or skin infection.

Study was conducted by cross-over style. 18 randomly assigned elderly healthy people or 18 elderly AD patients viewed a humorous film (The Best Bits of Mr. Bean, $72 \mathrm{~min}$, Universal studios, 1996) at 8:00 am. After 2 weeks, they viewed a control non-humorous film featuring weather information (72 $\mathrm{min}$ ) at 8:00 am. Alternatively, another $18 \mathrm{el}$ derly healthy people or 18 elderly AD patients first viewed a control film featuring weather information at 8:00 am, and after 2 weeks they viewed a humorous film (The Best Bits of Mr. Bean) at 8:00 am. Before and immediately after each viewing, saliva was collected and TEWL values on the back of the neck were measured by Tewameter $(\mathrm{C}+\mathrm{K}$ Co. Ltd., Kolen, Germany), as previously reported (18). TEWL values were registered in $\mathrm{g} / \mathrm{m}^{2} / \mathrm{h}$ after equilibration of the probe. Testosterone levels in fasting saliva were measured by EIA (Testosterone Kit, Oxford Biomedical Research, 
Oxford, MI, USA). As control, amylase levels in saliva were also measured by Kit $\alpha$-amylase Colorimetric Test (Diagnostica, Merck, Germany). Statistical analysis was performed with repeated measures of ANOVA. This study was approved by the Ethical Committee of Moriguchi-Keijinkai Hospital.

\section{Results}

As shown in Table 1A, viewing a control film had no effect on salivary testosterone levels, salivary amylase levels or TEWL values on the back of the neck in elderly healthy people. However, viewing a humorous film slightly, but significantly $(\mathrm{P}<0.05)$, elevated salivary testosterone levels and reduced TEWL values on the back of neck, while it did not elevate salivary amylase levels in_them. As shown in Table 1B, salivary testosterone levels at baseline (before viewing) were significantly decreased in elderly $\mathrm{AD}$ patients compared to those in elderly healthy people (156.1 $\mathrm{pg} / \mathrm{ml}$ vs. $209.4 \mathrm{pg} / \mathrm{ml}$ ), while salivary amylase levels were not significantly decreased (77.6 U/L vs. $78.4 \mathrm{U} / \mathrm{L})$. Moreover, TEWL values on the back of neck at the baseline were significantly $(\mathrm{P}<0.01)$ increased in elderly AD patients compared to those in elderly healthy people $\left(52.9 \mathrm{~g} / \mathrm{m}^{2} / \mathrm{h}\right.$ vs. $38.6 \mathrm{~g} / \mathrm{m}^{2} / \mathrm{h}$ ). Viewing a control film had no effect on salivary testosterone levels, salivary amylase levels or TEWL values on the back of neck in $\mathrm{AD}$ patients. In contrast, viewing a humorous film markedly $(\mathrm{P}<0.01)$ elevated salivary testosterone levels and reduced TEWL values on the back of the neck, while it did not elevate salivary amylase levels in $\mathrm{AD}$ patients.

\section{Discussion}

We demonstrated that viewing a humorous film markedly elevated salivary testosterone levels in elderly AD pa- tients, while viewing a control film did not do so. This was not due to non-specific elevation of protein in saliva, since salivary amylase levels were unchanged. There was a precedence for this. We previously reported that viewing a humorous film elevated breast milk leptin levels in lactating women with $\mathrm{AD}$ (15). Moreover, stress decreased testosterone levels (20). Thus, it was possible that relaxation by viewing a humorous film could elevate testosterone levels.

Moreover, viewing a humorous film reduced TEWL values in elderly AD patients. Reduction of TEWL values indicated that dryness of skin was improved. This was a novel finding, but was not surprising. Mental stress has a negative impact on skin barrier recovery in healthy humans, while relaxation by odorant inhalation alleviated stress-induced skin barrier dysfunction both in mice and humans $(11,19)$.

It was also reported that elevation of testosterone levels were associated with improvement in skin hydration and lipids $(8,12)$, while stress decreased testosterone levels and aggravated skin hydration (10). Collectively, it would be tempting to speculate that viewing a humorous film would elevate testosterone levels, which in turn might reduce TEWL values. On the other hand, the effect of other hormones, including growth hormones, should be considered, since growth hormones were involved in cutaneous parameters (6). In fact, viewing a humorous film elevated growth hormone levels in elderly AD patients (manuscript in preparation). Involvement of other hormones are currently under investigation.

Viewing a humorous film also slightly, but significantly, elevated salivary testosterone levels and reduced TEWL values in elderly people, while viewing a control film failed to do so. This was in accordance with a previous report that viewing a humorous film elevated breast milk leptin levels in healthy lactating women, while it augmented brachial vasodilatation in healthy people $(15,17)$. We also conducted

Tab. 1: Effect of viewing a humorous film on salivary testosterone and TEWL.

\begin{tabular}{|l|c|c|c|c|c|c|}
\hline & \multicolumn{2}{|c|}{ Control film } & \multicolumn{3}{c|}{ Humorous film } \\
\hline & Before & After & P & Before & After & P \\
\hline (A) Elderly healthy people & & & & & & \\
\hline Saliva & & & & & & \\
\hline Testosterone (pg/ml) & $209.4(38.4)$ & $204.3(33.1)$ & NS & $207.1(37.2)$ & $223.8(43.7)$ & $<0.05$ \\
\hline Amylase (U/L) & $78.4(31.2)$ & $74.1(27.0)$ & NS & $75.7(28.9)$ & $73.4(25.2)$ & NS \\
\hline Back of neck & & & & & & \\
\hline TEWL $(\mathrm{g} / \mathrm{m} 2 / \mathrm{h})$ & $38.6(13.8)$ & $40.2(16.3)$ & NS & $41.1(17.4)$ & $34.0(10.1)$ & $<0.05$ \\
\hline (B) Elderly patients with atopic dermatitis & & & & & & \\
\hline Saliva & & & & & & \\
\hline Testosterone $(\mathrm{pg} / \mathrm{ml})$ & $156.1(27.6)$ & $152.3(27.1)$ & $\mathrm{NS}$ & $150.5(25.9)$ & $191.7(37.2)$ & $<0.01$ \\
\hline Amylase $(\mathrm{U} / \mathrm{L})$ & $77.6(30.5)$ & $73.0(26.4)$ & $\mathrm{NS}$ & $76.2(27.4)$ & $72.9(25.8)$ & $\mathrm{NS}$ \\
\hline Back of neck & & & & & & \\
\hline TEWL $\left(\mathrm{g} / \mathrm{m}^{2} / \mathrm{h}\right)$ & $52.9(19.2)$ & $54.5(20.5)$ & $\mathrm{NS}$ & $54.7(22.8)$ & $42.0(17.3)$ & $<0.01$ \\
\hline
\end{tabular}

Thirty-six elderly healthy people (A) or 36 elderly patients with atopic dermatitis (B) viewed a control film or a humorous film. Before and after each viewing, levels of testosterone or amylase in saliva, and transepidermal water loss (TEWL) values of the back of neck were measured. Values are the means (SD). Statistical analysis was performed with ANOVA by comparing „before“ and „,after“. NS = not significant. 
a time-course study. The effect of viewing a humorous film was detectable after 3 hrs but not 4 hours. In 36 elderly AD patients, salivary testosterone levels (mean $[\mathrm{SD}] \mathrm{pg} / \mathrm{ml}$ ) were 150.5 [25.8] (before), 164.8 [28.7] (after 3 hours) $(\mathrm{p}<0.05)$ and 151.3 [26.5] (after 4 hours), while mean TEWL values (mean $[\mathrm{SD}] \mathrm{g} / \mathrm{m}^{2} / \mathrm{h}$ ) on the back of the neck were 54.7 [22.9] (before), 48.2[20.4] (after 3 hours) $(\mathrm{P}<0.05)$, and 54.1 [22.1] (after 4 hours). Thus, the positive effect of viewing a humorous film is short lasting. Taken together, viewing a humorous film may be helpful in improving various conditions in humans with or without diseases.

It should be noted that measurement of salivary testosterone levels and TEWL values are simple and non-invasive, thus it is useful in studing elderly people. Since dry skin was a risk factor for breaches in skin integrity, more research was needed to improve skin condition in elderly people (20). Our results suggest that viewing a humorous film may be useful in studies to improve dry skin in elderly people with or without $\mathrm{AD}$.

\section{References}

1. Baulieu EE, Thomas G, Legrain S et al. Dehydroepiandrosterone (DEHA) DHEA sulfate, and aging: contribution of the DHEAge Study to a sociobiomedical issue. Proc Natl Acad Sci USA 2000;97:4279-84.

2. Berardesca E, Fideli D, Borroni G, Rsbbiosi G, Msibsch H. In vivo hydration and water-retention capacity of stratum corneum in clinically uninvolved skin in atopic and psoriatic patients. Atca Derm Venereol 1990;70:400-4

3. Berg M, Arnetz BB, Liden S, Enerith P, Kallen A. Techno-stress. A psychological stuffy of employees with VDU-associated skin complaints. J Occup Med 1992;34:698-701.

4. Buske-Kirschbaum A, Geiben A, Hollig H, Morschhauser E, Hellhammer D Altered responsiveness of the hypothalamus-pituitary-adrenal axis and the sympathetic adrenomedullary system to stress in patients with atopic dermatitis. J Clin Endocrinol Metab 2002;87:4245-5
5. Choi EH, Brown BE, Crumrine D et al. Mechanisms by which psychologic stress alters cutaneous permeability barrier homeostasis and stratum corneum integrity. J Invest Dermatol 2005;124:587-95.

6. Conte F, Diridollou S, Jouret B et al. Evaluation of cutaneous modifications seventy-seven growth hormone-deficient children. Horm res 2000;54:92-7.

7. Cua AB, Wilheim KP, Maibach HI. Erictional properties of human skin: relation to age, sex and anatomical region, stratum croneum hydration and transepidermal water loss. Br J Dermatol 1990;123:473-9.

8. Denda M, Tsuchiya T, Shoji K, Tanida M. Odorant inhalation affects skin barrie homeostasis in mice and humans. Brit J Dermatol 2000;142:1007-10.

9. Ebata T, Itamura R, Aizawa H, Niimura M. Serum sex hormone levels in adult patients with atopic dermatitis. J Dermatol 1996;23:603-5.

10. Hodgkinson B, Nay R, Wilson J. A systematic review of topical skin care in aged care facilities. J Clin Nurs 2007;16:129-36.

11. Hosoi J, Tanida M, Tsuchiya T. Mitigation of stress-induced suppression of contact hypersensitivity by odorant inhalation. Br J Dermatol 2001;145:716-9.

12. Imaeda K, Ohsawa K, Watanabe T. Effect of sex-hormone on composition of rat skin surface lipid. Nippon Yakugaku Zasshi 1987;90:147-53.

13. Kimata H. Enhancement of allergic skin wheal responses and in vitro allergenspecific IgE production by computer-induced stress in patients with atopic dermatitis. Brain Behav Immun 2003;17:134-8.

14. Kimata H. Laughter counteracts enhancement of plasma neurotrophin levels and allergic skin wheal responses by mobile phone-mediated stress. Physiol Behav 2004;81:681-4

15. Kimata H. Elevation of breast milk leptin levels by laughter. Horm Metab Res 2004;36:254-6

16. Kunz B, Oranje AP, Labreza L, Stalder J-F, Ring J, Taieb A. Clinical validation and guidelines for the SCORAD index: Consensus report of the European Task Force on atopic dermatitis. Dermatology 1997;195:10-9

17. Miller M, Mangano C, Park Y, Goel R, Plotnick GD, Vogel RA. Impact of cinematic viewing on endothelia function. Heart 2006;92:261-2.

18. Miyanishi K, Shiono N, Shirai H, Dombo M, Kimata H. Reduction of transepidermal water loss by oral intake of glucosylceramides in patients with atopic eczema. Allergy 2005;60:1454-5.

19. Muizzuddin N, Matui MS, Marenus KD, Maes DH. Impact of stress of martial dissolution on skin barrier recovery: tape stripping and measurement of transepidermal water loss (TEWL). Skin Res Technol 2003;9:34-8.

20. Rev F, Chiodoni G, Gomez F, Felber JP. Interpretation of the discrepancy observed between plasma free and salivary testosterone levels in man. Steroids 1988;52:371-2.

21. Tsuchiya T, Horii I. Immobilization-induced stress decreases lipogenesis in sebaceous glands as well as plasma testosterone levels in male Syrian hamsters. Psychoneuroendocrinology 1995;20:221-30.

Submitted January 2007. Accepted April 2007.

\section{Corresponding author:}

Hajime Kimata, MD., Ph.D., Department of Allergy, Moriguchi-Keijinkai Hospital, 2-47-12, Yagumo-Higashimachi, Moriguchi City, Osaka Prefecture, 570-0021, Japan, e-mail: kimata-keijinkai@mkc.zaq.ne.jp 